\title{
Layer cake: the first bite at gene expression diversity in the human brain
}

\author{
Philipp Khaitovich ${ }^{1,2, *}$
}

\begin{abstract}
A recent publication has provided a comprehensive atlas of gene expression profiles of 995 genes linked to neuronal functions in two regions of the human brain neocortex.
\end{abstract}

The human brain is a remarkably complex organ that provides our species with a unique set of cognitive abilities. What kind of molecular mechanisms determine this functional uniqueness are currently unknown. This is at least partially due to the sheer intimidating complexity of the human brain: most studies conducted to date have preferred to focus on simpler biological systems. As a result, we now have plenty of information about gene expression profiles in each of the 959 somatic cells constituting the roundworm Caenorhabditis elegans [1]. At the same time, we know almost nothing about gene expression variation among cells constituting the human brain. A recent publication in Cell by Allan Jones and colleagues aims to break this stalemate, providing researchers with a comprehensive atlas of gene expression profiles of 995 genes linked to neuronal functions in two regions of the human brain neocortex [2].

The neocortexis is the evolutionarily most recent part of the human brain that forms the top layer of cerebral hemispheres, and is present in mammals but not in birds or reptiles. The neocortex is normally 2 to $5 \mathrm{~mm}$ thick, and contains most of the cortical neurons. Within the neocortex, neurons and their projections are not distributed uniformly, but form a distinct laminar sixlayer architecture. These layers, historically labeled I to VI, with VI being the innermost and I being the outermost, are conserved across mammals and are largely recognizable in all regions of the neocortex. Different

*Correspondence: khaitovich@eva.mpg.de

${ }^{2}$ Max Planck Institute for Evolutionary Anthropology, Deutscher Platz 6, Leipzig,

04103, Germany

Full list of author information is available at the end of the article parts of human hemispheres vary in thickness and neuronal density of individual cortical layers. This variability in layer architecture constitutes the basis of cortical region classification, or cytoarchitectonic cortical maps; the one most commonly used today was created by Korbinian Brodmann more than a century ago [3]. More recently, whole transcriptome profiling using microarrays demonstrated the presence of distinct expression patterns characteristic of specific cortical regions in the human brain $[4,5]$. These studies, however, were conducted on tissue samples containing a mixture of cortical layers and, therefore, did not contain information of layer-specific expression variation across cortical regions.

Previous studies that aimed to elucidate gene expression patterns characteristic of individual cortical layers were conducted mainly in mice. Gene expression profiling of mouse cortical layers conducted using microarrays [6,7] or high-throughput sequencing [7] have indicated that thousands of genes show distinct expression patterns across neocortical layers. Furthermore, layer-specific expression of hundreds of genes was shown in the mouse brain by in situ hybridization (ISH) with probes complementary to specific mRNA within the framework of the Allen Mouse Brain Atlas project [8]. The presence of clear, layer-specific gene expression signatures is not unexpected, since different layers comprise different cell types, and cell types are determined by their gene expression. To determine the relevance of these findings to the human brain, it would be important to know how many of the layer-specific expression patterns found in the mouse brain are present in humans and what layerspecific expression features have evolved on the human evolutionary lineage.

\section{Study details}

To address this question, Allan Jones and colleagues used ISH to assess the location and expression intensity of 995 genes linked to neural functions. The researchers focused on two distinct neocortical regions: the rear part of the occipital lobe located at the back of the brain, and the middle part of the temporal lobe located at the side of the brain. The occipital lobe region contained Brodmann's area 17 (a primary visual cortex area receiving the signal 
from the optical nerve) and Brodmann's area 18 (a secondary visual cortex area involved in visual signal processing). A mid-temporal region mostly contained Brodmann's area 21 implicated in a number of functions, including facial recognition and distance assessment. Based on approximately 31,000 ISH images generated in the study, the researchers were able to identify layerspecific and broadly expressed genes in each of these areas. Using these data, researchers asked whether gene expression across layers varies among individuals, whether it differs among Brodmann's areas, and whether it is conserved between corresponding areas of the human and mouse brain.

Among genes with detectable ISH signal, 95\% showed a consistent expression pattern across individuals. Still, given the relatively small number of individuals tested, an average of four for each marker, it is notable that as many as $5 \%$ of genes showed clear individual differences in expression localization and intensity. While sounding farfetched at present, this type of analysis might one day result in the identification of gene expression variation associated with individual personality traits.

Perhaps more surprisingly, researchers found that layer-specific expression is largely conserved among distant brain regions. Thus, only $4 \%$ of genes showed substantial differences between Brodmann's areas 18 and 21 located in occipital and temporal hemispheric lobes, respectively. In contrast, the primary visual cortex (Brodmann's area 17) showed a quite distinct expression profile from neighboring area 18, with $15 \%$ of genes showing an expression pattern change across the area boundary. These results fit well into observations obtained by a broader study of gene expression in rhesus macaque cortical layers in ten discrete areas of rhesus macaque cortex conducted using microarrays [9]. The cortical areas examined in that study spanned four primary sensorimotor cortices, including primary visual cortex, three higher-order visual areas, and three frontal cortical areas. The analysis, based on hybridization signals from more than 15,000 array probes, has shown that gene expression signatures of the cortical layers are remarkably similar across all regions, with the sole expectation of the primary visual cortex (Brodmann's area 17). Unexpectedly, expression profiles across cortical layers in the ten brain regions clustered based on their special proximity within brain rather than on their function. Thus, layer architecture of the cortical areas might be determined by developmental programs' relaying of the special gradients of regulatory factors, rather than by assignment of these areas to specific functions.

More insights have come from an evolutionary comparison of layer-specific expression patterns observed by Allan Jones and colleagues in the human and expression of the corresponding genes in the mouse brain [8].
Approximately 25\% of examined genes showed substantial differences in special expression patterns across layers between humans and mice, with the vast majority of these differences being consistent between two brain regions. Interestingly, most of these differences (68\%) affected genes showing distinct layer-specific profiles, rather than genes broadly expressed within cortex. One of the most obvious differences separating humans and mice was the loss of a specific expression pattern in layer $\mathrm{V}$ in the human cortex. Interestingly, some of the genes forming this pattern shifted their expression from layer $\mathrm{V}$ neurons in mouse to layer III neurons in human. Layer V neurons mainly send their output projections to subcortical and contralateral regions, while layer III neurons mainly form intracortical connections. Layers II and III are expanded in humans and other primates compared with the mouse, suggesting substantially increased intracortical connectivity in the primate brain. Thus, a shift in expression specificity from layer $\mathrm{V}$ in mice to layer III in humans might reflect a need for further neuronal specialization in the cortical layer involved in long-range intracortical connectivity.

\section{Conclusion}

From the molecular biology perspective, the human brain still represents a largely unexplored territory. The difficulties faced by scientists studying the human brain are many: getting good tissue samples, an inability to conduct genetic and functional experiments in humans, and finally difficulty in obtaining a comprehensive picture encompassing the entire brain. Nevertheless, it is studies focusing on the identification of molecular features that separate our brains from brains from other species that hold the most promise to deliver the answer to one of the most exiting questions of biology: what makes us so different from other species?

The study conducted by Allan Jones and colleagues [2], as well as the transcriptome profiling in the macaque brain conducted by Ed Lein and colleagues [9], represent the important first steps in this direction. The results are encouraging: although a limited number of genes and cortical regions were examined in the Jones study, several substantial transitions in layer-specific expression patterns between humans and mice, affecting as much as a quarter of all examined genes, could be identified. Differences in the expression localization affect distribution of ion channels, cell adhesion molecules, as well as other cellular components involved in neural signal transduction and cell-cell communication. This shows that despite overall similarities of cellular architecture between the human and mouse neocortex, there are substantial differences in molecular and potentially functional composition of cortical layers between the two species. Similarly, results obtained by Ed Lein and colleagues show much 
greater agreement in cortical layer expression between humans and macaques than between these primate species and mice. Those results indicate that studies of human brain functionality might still require the use of non-human primates as a model system until other alternatives, such as artificially grown human tissue, are available.

Taken together, these results show that comprehensive molecular studies of the human brain can provide deep and meaningful insights into the nature of molecular mechanisms underlying human-specific cognitive abilities. Further studies comparing gene expression profiles in distinct cortical structures between humans and closely related primate species, such as chimpanzees and macaques, can provide further insights into molecular mechanisms specific to the human brain. To obtain biologically sound results, such studies might need to include different stages of brain development and maturation in addition to adult brain samples [10].

Abbreviation

ISH, in situ hybridization.

\section{Competing interests}

The author declares that there are no competing interests.

\section{Author details}

'CAS-MPG Partner Institute for Computational Biology, Yue Yang road 320, Shanghai, 200031, China. ${ }^{2}$ Max Planck Institute for Evolutionary Anthropology, Deutscher Platz 6, Leipzig, 04103, Germany.

Published: 22 May 2012

\section{References}

1. Liu X, Long F, Peng H, Aerni SJ, Jiang M, Sanchez-Blanco A, Murray J, Preston E, Mericle B, Batzoglou S, Myers EW, Kim SK: Analysis of cell fate from singlecell gene expression profiles in C. elegans. Cell 2009, 139:623-633.

2. Zeng H, Shen EH, Hohmann JG, Oh SW, Bernard A, Royall JJ, Glattfelder KJ, Sunkin SM, Morris JA, Guillozet-Bongaarts AL, Smith KA, Ebbert AJ, Swanson
B, Kuan L, Page DT, Overly CC, Lein ES, Hawrylycz MJ, Hof PR, Hyde TM, Kleinman JE, Jones AR: Large-scale cellular-resolution gene profiling in human neocortex reveals species-specific molecular signatures. Cell 2012, 149:483-496.

3. Brodmann K: Vergleichende Lokalisationslehre der Großhirnrinde in ihren Prinzipien dargestellt auf Grund des Zellenbaues. Leipzig: Johann Ambrosius Barth Verlag; 1909

4. Khaitovich P, Muetzel B, She X, Lachmann M, Hellmann I, Dietzsch J, Steigele S, Do H, Weiss G, Enard W, Heissig F, Arendt T, Nieselt-Struwe K, Eichler EE, Pääbo S: Regional patterns of gene expression in human and chimpanzee brains. Genome Res 2004, 14:1462-1473.

5. Kang HJ, Kawasawa Yl, Cheng F, Zhu Y, Xu X, Li M, Sousa AM, Pletikos M, Meyer KA, Sedmak G, Guennel T, Shin Y, Johnson MB, Krsnik Z, Mayer S, Fertuzinhos S, Umlauf S, Lisgo SN, Vortmeyer A, Weinberger DR, Mane S, Hyde TM, Huttner A, Reimers M, Kleinman JE, Sestan N: Spatio-temporal transcriptome of the human brain. Nature 2011, 478:483-489.

6. Wang WZ, Oeschger FM, Lee S, Molnar Z: High quality RNA from multiple brain regions simultaneously acquired by laser capture microdissection. BMC Mol Biol 2009, 10:69.

7. Belgard TG, Marques AC, Oliver PL, Abaan HO, Sirey TM, Hoerder-Suabedissen A, Garcia-Moreno F, Molnar Z, Margulies EH, Ponting CP: A transcriptomic atlas of mouse neocortical layers. Neuron 2011, 71:605-616.

8. Lein ES, Hawrylycz MJ, Ao N, Ayres M, Bensinger A, Bernard A, Boe AF, Boguski MS, Brockway KS, Byrnes EJ, Chen L, Chen L, Chen TM, Chin MC, Chong J, Crook BE, Czaplinska A, Dang CN, Datta S, Dee NR, Desaki AL, Desta T, Diep E, Dolbeare TA, Donelan MJ, Dong HW, Dougherty JG, Duncan BJ, Ebbert AJ, Eichele G: Genome-wide atlas of gene expression in the adult mouse brain. Nature 2007, 445:168-176.

9. Bernard A, Lubbers LS, Tanis KQ, Luo R, Podtelezhnikov AA, Finney EM, McWhorter MM, Serikawa K, Lemon T, Morgan R, Copeland C, Smith K, Cullen V, Davis-Turak J, Lee CK, Sunkin SM, Loboda AP, Levine DM, Stone DJ, Hawrylycz MJ, Roberts CJ, Jones AR, Geschwind DH, Lein ES: Transcriptional architecture of the primate neocortex. Neuron 2012, 73:1083-1099.

10. Somel M, Liu X, Tang L, Yan Z, Hu H, Guo S, Jiang X, Zhang X, Xu G, Xie G, Li N, Hu Y, Chen W, Pääbo S, Khaitovich P: MicroRNA-driven developmental remodeling in the brain distinguishes humans from other primates. PLOS Biol 2011, 9:e1001214.

doi:10.1186/gb-2012-13-5-157

Cite this article as: Khaitovich P: Layer cake: the first bite at gene expression diversity in the human brain. Genome Biology 2012, 13:157. 